Supporting Information

\title{
Molecular, Seasonal and Spatial Distributions of Organic Aerosols from Fourteen Chinese Cities
}

\author{
GEHUI WANG $^{\dagger, \ddagger}$ AND KIMITAKA KAWAMURA ${ }^{\dagger, *}$, \\ ${ }^{\dagger}$ Institute of Low Temperature Science, Hokkaido University, Kita 19, Nishi 8, Kita-ku, \\ Sapporo 060-0819, Japan \\ ${ }^{\ddagger}$ School of the Environment, State Key Laboratory of Pollution Control and Resources \\ Reuse, Nanjing University, Nanjing 210093, China
}

SHUNCHENG LEE AND KINFAI HO

Research Center for Environmental Technology and Management, Department of Civil and Structural Engineering, Hong Kong Polytechnic University, Hong Kong, China

\section{JUNJI CAO}

SKLLQG, Institute of Earth Environment, Chinese Academy of Sciences, Xi'an, China

*Corresponding author phone: 81-11-706-5457; fax: 81-11-706-7142, e-mail:

kawamura@lowtem.hokudai.ac.jp

Revised version submitted to Environmental Science \& Technology

On April 24, 2006

Summary of the supporting information:

(1) The number of pages of the supporting information is 6 , including one table and one figure.

(2) Table S-1. Concentrations of individual organic compounds in aerosols from 14 Cities of China

(3) Figure S-1. Salient features of the GC/MS traces for the minor compounds in aerosols from Xi'an during winter $(01 / 13 / 2003)$ (a) mass chromatogram ( $\mathrm{m} / \mathrm{z} 75)$ of fatty alcohols, (b) mass chromatogram ( $\mathrm{m} / \mathrm{z} 147)$ of polyols and polyacids, (c) mass chromatogram (m/z 129) of sterols, and (d) mass chromatogram (m/z 191) of hopanes. 


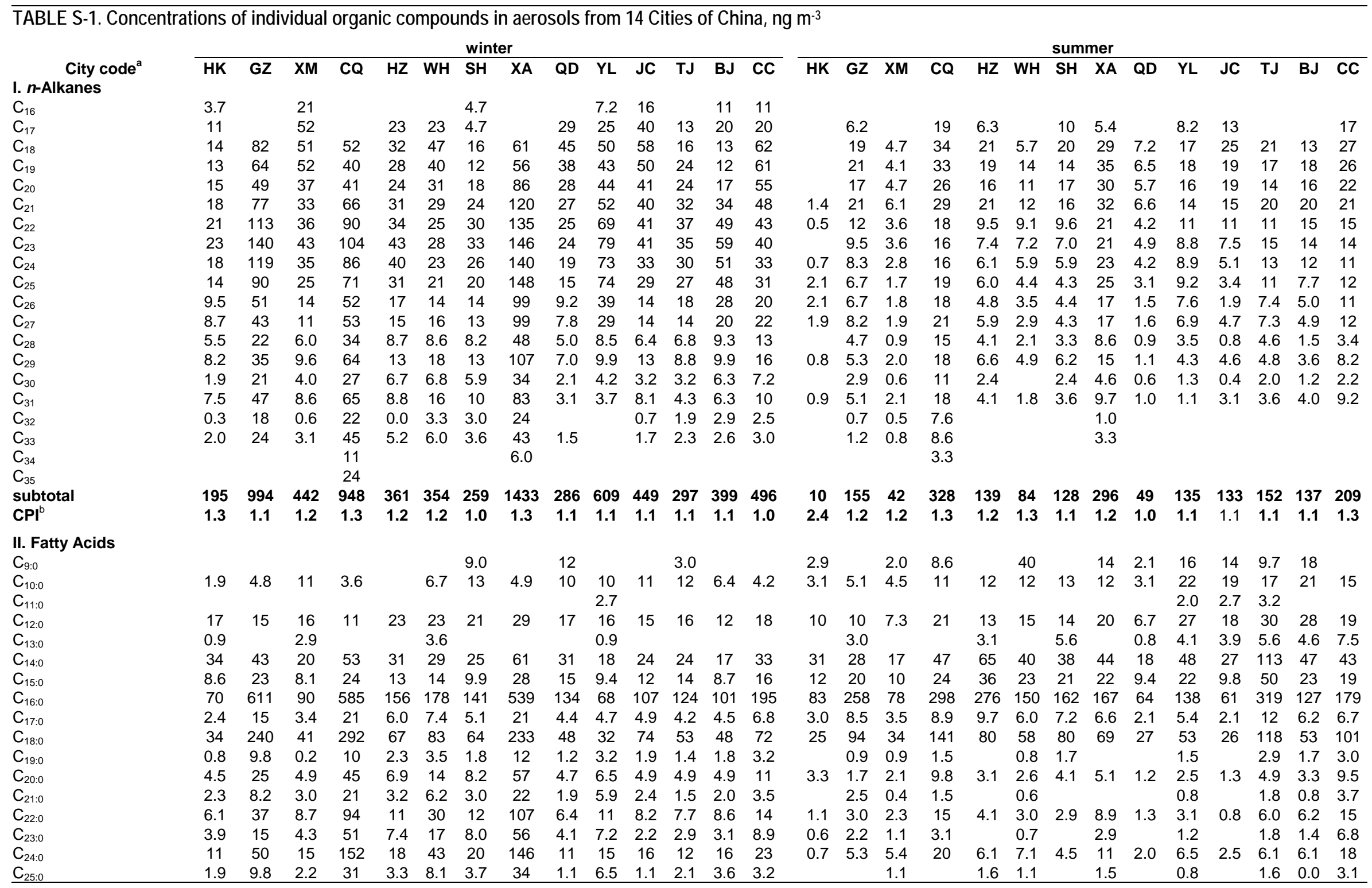




\begin{tabular}{|c|c|c|c|c|c|c|c|c|c|c|c|c|c|c|c|c|c|c|c|c|c|c|c|c|c|c|c|c|}
\hline \multicolumn{29}{|c|}{ TABLE S-1 (Continued). } \\
\hline & HK & GZ & $\mathrm{XM}$ & CQ & $\mathrm{HZ}$ & WH & $\mathrm{SH}$ & $\mathrm{XA}$ & QD & YL & $\mathrm{JC}$ & TJ & BJ & $\mathrm{CC}$ & HK & GZ & $\mathrm{XM}$ & CQ & $\mathrm{HZ}$ & WH & SH & $\mathrm{XA}$ & QD & YL & $\mathrm{JC}$ & TJ & BJ & CC \\
\hline $\mathrm{C}_{26: 0}$ & 6.8 & 31 & 5.6 & 80 & 10 & 25 & 8.6 & 74 & 7.3 & 16 & 13 & 8.7 & 14 & 9.3 & & 1.8 & 3.1 & 10 & & 4.3 & 3.8 & 3.7 & & 1.9 & & 3.7 & 3.6 & 6.2 \\
\hline $\mathrm{C}_{27.0}$ & & 3.7 & & 17 & & 3.8 & & 14 & & 3.3 & & & & & & & 0.7 & & & & & & & & & & & \\
\hline $\mathrm{C}_{28: 0}$ & 8.4 & 42 & 9.4 & 105 & 11 & 24 & 10 & 97 & 4.0 & 8.4 & 8.3 & 5.3 & 8.0 & 9.0 & & 4.4 & 4.9 & 11 & & 4.5 & 3.7 & 8.2 & & 2.7 & & 2.5 & & 4.1 \\
\hline $\mathrm{C}_{29: 0}$ & 0.8 & & & 3 & & 6.9 & & 1.1 & & & & & & & & & & & & & & & & & & & & \\
\hline $\mathrm{C}_{30: 0}$ & 9.1 & 48 & 11 & 221 & 13 & 36 & 17 & 106 & 5.3 & & & & 4.6 & 13 & & 6.0 & 7.8 & 5.7 & & 10 & & & & & & 2.6 & & \\
\hline $\mathrm{C}_{31: 0}$ & & & & 30 & & & & & & & & & & & & & & & & & & & & & & & & \\
\hline $\mathrm{C}_{32: 0}$ & & 32 & & 132 & & 20 & 6.5 & 42 & & & & & & & & & & & & & & & & & & & & \\
\hline $\mathrm{C}_{34: 0}$ & & & & 37 & & & & & & & & & & & & & & & & & & & & & & & & \\
\hline$C_{16: 1}$ & 58 & 188 & 36 & 52 & 84 & 101 & 58 & 147 & 158 & 80 & 111 & 93 & 52 & 120 & 31 & 34 & 50 & 96 & 57 & 66 & 50 & 47 & 12 & 104 & 39 & 87 & 80 & 27 \\
\hline $\mathrm{C}_{18: 1}$ & 34 & 1767 & 40 & 1141 & 161 & 92 & 55 & 1029 & 92 & 78 & 77 & 78 & 97 & 126 & 22 & 10 & 31 & 103 & 20 & 37 & 15 & 25 & 5.4 & 109 & 17 & 76 & 39 & 22 \\
\hline $\mathrm{C}_{20: 1}$ & & & & 7.7 & & & & & & & & & & & & & & & & & & & & & & & & \\
\hline $\mathrm{C}_{24: 1}$ & 1.1 & & & & & & & & & & & & & & & & & & & & & & & & & & & \\
\hline subtotal & 318 & 3219 & 333 & 3244 & 626 & 774 & 501 & 2859 & 567 & 402 & 495 & 468 & 413 & 687 & 229 & 498 & 266 & 837 & 585 & 482 & 425 & 467 & 155 & 572 & 245 & 877 & 472 & 509 \\
\hline $\mathrm{CPI}^{\mathrm{b}}$ & 11 & 14 & 9.7 & 7.4 & 10 & 7.2 & 8.1 & 8.0 & 7.2 & 4.7 & 12 & 9.4 & 14 & 10 & 8.5 & 11 & 8.4 & 13 & 9.6 & 4.8 & 9.1 & 7.6 & 8.3 & 5.7 & 4.8 & 7.1 & 5.4 & 8.9 \\
\hline \multicolumn{29}{|l|}{ III. Sugars } \\
\hline levoglucosan & 130 & 848 & 132 & 2706 & 260 & 582 & 455 & 3204 & 156 & 63 & 166 & 196 & 171 & 329 & 6.2 & 49 & 16 & 690 & 59 & 66 & 37 & 158 & 16 & 26 & 30 & 109 & 41 & 222 \\
\hline arabitol & 1.4 & & & 12 & & & 3.9 & & & & & & & & & 0.5 & & & & & & 0.9 & & 0.8 & 0.9 & & & 5.3 \\
\hline fructose & 1.4 & 55 & & 4.2 & & & & & & & & & 5.0 & 1.5 & & 1.4 & & 11 & 1.4 & & 1.1 & 4.6 & & 6.8 & 1.5 & 2.8 & & 16 \\
\hline glucose & 12 & 30 & 5.3 & 43 & 7.9 & 17 & 8.3 & 26 & 3.4 & 1.0 & 2.5 & 6.1 & 6.9 & 8.0 & 2.4 & 4.8 & 1.7 & 24 & 3.8 & 2.8 & 2.9 & 11 & 0.9 & 2.4 & 3.4 & 7.2 & 2.7 & 13 \\
\hline mannitol & 1.1 & & 0.6 & & & & & & & & & & & & & 0.5 & & & & & & & & 0.5 & 0.7 & 2.1 & & 3.3 \\
\hline inositol & 0.8 & 21 & 1.9 & 29 & 3.7 & 5.7 & 3.2 & 8.9 & 1.1 & & 0.3 & 1.0 & 1.5 & 1.0 & 0.2 & 0.1 & & 6.4 & 0.8 & & 0.3 & 3.0 & 0.2 & 0.6 & 0.6 & 1.2 & & 4.9 \\
\hline sucrose & 1.6 & 16 & 1.1 & 4.1 & 3.2 & 4.3 & 2.9 & & 0.7 & & 0.8 & 2.0 & 14 & 3.2 & 0.6 & 1.9 & & 3.1 & 1.0 & 0.7 & 0.7 & 2.3 & & 2.2 & 1.3 & 1.1 & 0.4 & 5.3 \\
\hline trehalose & 0.4 & & 0.3 & & & & & & & & 0.4 & & & & 0.1 & 0.9 & & 0.6 & 0.5 & 0.4 & & 2.5 & & 0.8 & 2.0 & 1.3 & 0.3 & 4.7 \\
\hline subtotal & 148 & 969 & 141 & 2799 & 275 & 609 & 473 & 3239 & 161 & 64 & 170 & 205 & 198 & 342 & 9.4 & 59 & 18 & 735 & 66 & 70 & 42 & 182 & 17 & 40 & 41 & 125 & 45 & 275 \\
\hline \multicolumn{29}{|l|}{ IV. Phthalates } \\
\hline dimethyl & 2.9 & 0.5 & 3.9 & 0.9 & 5.1 & 1.9 & 1.9 & 0.3 & 2.6 & 1.5 & 2.1 & 1.3 & 1.5 & 1.9 & 0.8 & 1.1 & 0.4 & 1.0 & 1.1 & 1.3 & 1.4 & 1.6 & 0.7 & 1.3 & 2.1 & 0.9 & 1.7 & 2.2 \\
\hline diethyl & 12 & 1.1 & 7.1 & 2.1 & 5.4 & 5.4 & 5.1 & 5.6 & 4.7 & 4.1 & 3.9 & 3.9 & 3.5 & 4.3 & 6.6 & 3.8 & 2.1 & 5.3 & 6.8 & 6.6 & 13 & 14 & 4.1 & 23 & 14 & 9.0 & 11 & 15 \\
\hline diisobutyl & 20 & 31 & 30 & 35 & 45 & 37 & 43 & 79 & 19 & 17 & 23 & 24 & 22 & 28 & 36 & 104 & 43 & 175 & 137 & 130 & 164 & 236 & 44 & 57 & 64 & 281 & 122 & 118 \\
\hline di-n-butyl & 42 & 37 & 31 & 53 & 34 & 29 & 32 & 48 & 28 & 14 & 19 & 21 & 21 & 18 & 117 & 121 & 52 & 270 & 165 & 131 & 158 & 185 & 64 & 60 & 97 & 269 & 135 & 143 \\
\hline benzylbutyl & & & & & & & & & & & & & & & 0.8 & & 2.2 & 1.9 & & 2.6 & & 1.1 & & & & 1.2 & 2.6 & \\
\hline bis(2-ethylhexyl) & 96 & 262 & 76 & 244 & 115 & 63 & 258 & 313 & 44 & 25 & 39 & 75 & 130 & 63 & 241 & 202 & 102 & 1748 & 266 & 198 & 277 & 225 & 55 & 119 & 110 & 394 & 130 & 133 \\
\hline subtotal & 172 & 332 & 148 & 335 & 204 & 136 & 341 & 445 & 99 & 62 & 87 & 126 & 178 & 115 & 403 & 432 & 201 & 2201 & 576 & 470 & 613 & 663 & 168 & 261 & 288 & 956 & 402 & 412 \\
\hline \multicolumn{29}{|l|}{ V. Fatty Alcohols } \\
\hline $\mathrm{C}_{12}$ & & & & & & & 13 & & & & & & & & & & & & & & 0.0 & 3.7 & 0.9 & 12 & & & & \\
\hline $\mathrm{C}_{14}$ & 3.2 & & & & & & & & & & & & & & & & & & & & 5.5 & 4.8 & 2.0 & 7.4 & 14 & 6.4 & & 7.6 \\
\hline $\mathrm{C}_{16}$ & 9.7 & 11.3 & 4.8 & 8.6 & 4.4 & 7.4 & 7.7 & 16 & 5.4 & 4.4 & 2.8 & 2.7 & 1.2 & 5.3 & 4.4 & 1.3 & 2.5 & 10 & 8.6 & 9.2 & 9.3 & 14 & 3.5 & 8.4 & 10 & 9.9 & 6.5 & 8.2 \\
\hline $\mathrm{C}_{18}$ & 9.0 & 13.2 & 7.8 & 13 & 7.3 & 6.8 & 9.7 & 18 & 6.6 & 4.1 & 5.3 & 3.3 & 3.1 & 7.0 & 7.7 & 1.8 & 4.2 & 11 & 10 & 11 & 4.6 & 20 & 3.5 & 10.0 & 4.5 & 11 & 5.5 & 4.6 \\
\hline $\mathrm{C}_{20}$ & & & & 16 & & & & 16 & & & & & & & & & & & 1.8 & & & 2.8 & & & & & & 4.0 \\
\hline
\end{tabular}




\section{TABLE S-1 (Continued)}

$\mathrm{C}_{22}$

$\mathrm{C}_{24}$

$\mathrm{C}_{26}$
$\mathrm{C}_{28}$

$\mathrm{C}_{30}$

$\mathrm{C}_{32}$

subtotal

VI. Polyols and Polyacids

glycerol

glyceric acid

malic acid

tartaric acid

subtotal

VII. Lignin and Resin Products

vanillic acid

syringic acid

dehydroabietic acid

subtotal

\section{Sterols}

cholesterol

ergosterol

stigmasterol

$\beta$-sitosterol

subtotal

IX. PAHs

phenanthrene

anthracene

fluoranthene

pyrene

benzo(b)fluorene

benz(a)anthracene

chrysene/triphenylene

benzo(b)fluoranthene

benzo $(k)$ fluoranthene

benzo(e)pyrene

benzo(a)pyrene

perylene

indeno $(1,2,3-c d)$ pyrene

dibenz $(a, h)$ anthracene winter

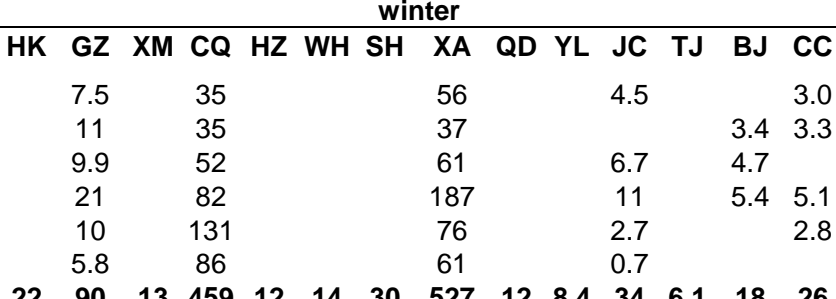

$\begin{array}{llllllllllllll}22 & 90 & 13 & 459 & 12 & 14 & 30 & 527 & 12 & 8.4 & 34 & 6.1 & 18 & 26\end{array}$

$\begin{array}{llllllllllllll}48 & 63 & 32 & 146 & 36 & 57 & 70 & 200 & 45 & 17 & 24 & 26 & 20 & 51\end{array}$ $\begin{array}{llllllllllllll}18 & 83 & 48 & 180 & 26 & 52 & 24 & 75 & 13 & 8.7 & 7.0 & 7.1 & 5.8 & 22\end{array}$ $\begin{array}{llllllllllllll}38 & 31 & 23 & 113 & 19 & 43 & 18 & 29 & 5.5 & 11 & 4.8 & 6.7 & 6.8 & 10\end{array}$

$\begin{array}{llllllllllllll}105 & 177 & 103 & 439 & 81 & 152 & 111 & 304 & 63 & 37 & 36 & 40 & 33 & 84\end{array}$

$\begin{array}{llllllllllllll}0.8 & 20 & 2.0 & 43 & 5.2 & 11 & 6.8 & 58 & 4.2 & 3.4 & 6.4 & 4.1 & 3.8 & 13\end{array}$ $\begin{array}{llllllllllllll}0.2 & 13 & 0.7 & 18 & 2.0 & 4.2 & 3.1 & 39 & 0.7 & 2.2 & 2.8 & 1.6 & 2.5 & 7.4\end{array}$ $\begin{array}{llllllllllllll}3.7 & 162 & 9.2 & 84 & 16 & 28 & 22 & 236 & 38 & 2.8 & 6.4 & 8.5 & 12 & 15\end{array}$ $\begin{array}{llllllllllllll}4.8 & 195 & 12 & 145 & 24 & 43 & 32 & 333 & 43 & 8.4 & 16 & 14 & 18 & 36\end{array}$

$\begin{array}{llllllllllllll}7.0 & 103 & 11 & 46 & 78 & 11 & 11 & 36 & 11 & 11 & 15 & 14 & 23 & 15\end{array}$

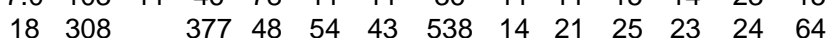

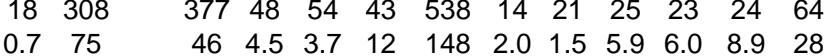
$\begin{array}{ccccccccccccc}0.7 & 75 & 46 & 4.5 & 3.7 & 12 & 148 & 2.0 & 1.5 & 5.9 & 6.0 & 8.9 & 28 \\ 0.3 & 438 & 261 & 27 & 20 & 26 & 729 & 8.8 & 452 & 27 & 25 & 32 & 101\end{array}$ $\begin{array}{lllllllllllllll}26 & 924 & 11 & 729 & 157 & 88 & 92 & 1451 & 36 & 485 & 72 & 67 & 87 & 207\end{array}$

$\begin{array}{llllllllllllll}1.9 & 24.5 & 4.1 & 8.1 & 3.5 & 3.1 & 3.8 & 9.4 & 2.4 & 2.8 & 2.0 & 2.3 & 0.9 & 2.2\end{array}$ 0.1

$\begin{array}{llllllllllllll}1.6 & 3.8 & 0.7 & 12 & 1.7 & 4.4 & 3.3 & 29 & 3.8 & 14 & 6.0 & 7.7 & 11 & 15\end{array}$ $\begin{array}{llllllllllllll}1.8 & 6.2 & 0.8 & 15 & 1.1 & 5.0 & 3.4 & 34 & 4.2 & 14 & 7.4 & 8.5 & 12 & 18\end{array}$ $\begin{array}{lllllllllllll}0.0 & 5.3 & 13 & 0.0 & 1.4 & 0.5 & 22 & 1.7 & 8.5 & 9.2 & 4.0 & 11 & 8.0\end{array}$ $\begin{array}{lllllllllllllll}0.3 & 12 & 0.1 & 31 & 0.6 & 2.0 & 2.0 & 42 & 2.1 & 15 & 11 & 9.0 & 19 & 13\end{array}$ $\begin{array}{lllllllllllllll}0.5 & 17 & 0.2 & 33 & 0.7 & 4.3 & 3.2 & 44 & 4.3 & 10 & 16 & 9.4 & 1 & 9.6\end{array}$ $\begin{array}{lllllllllllllll}3.5 & 83 & 7.9 & 168 & 16 & 35 & 25 & 187 & 34 & 51 & 62 & 36 & 59 & 49\end{array}$ $\begin{array}{llllllllllllll}3.6 & 83 & 7.9 & 168 & 16 & 35 & 25 & 187 & 34 & 51 & 62 & 36 & 59 & 49 \\ 0.7 & 15 & 1.4 & 21 & 1.9 & 6.4 & 4.5 & 29 & 2.3 & 9.3 & 9.0 & 7.0 & 8.3 & 9.3\end{array}$ $\begin{array}{llllllllllllll}0.9 & 18 & 1.9 & 40 & 3.7 & 11 & 5.9 & 41 & 6.6 & 9.9 & 18 & 7.7 & 12 & 9.6\end{array}$ $\begin{array}{llllllllllllll}0.7 & 24 & 1.1 & 33 & 1.7 & 4.6 & 4.4 & 47 & 3.9 & 12 & 9.5 & 7.1 & 14 & 12\end{array}$ $\begin{array}{llllllllllllll}0.2 & 5.4 & 0.2 & 6.5 & 0.8 & 1.3 & 1.3 & 12 & 0.7 & 2.7 & 2.1 & 2.0 & 3.7 & 3.1\end{array}$ $\begin{array}{llllllllllllllll}0.7 & 38 & 2.8 & 52 & 5.7 & 12 & 8.5 & 67 & 6.6 & 17 & 16 & 11 & 15.2 & 17\end{array}$ $\begin{array}{llllllllllll}0.3 & 4.9 & 9.0 & 1.9 & 0.8 & 12 & 1.0 & 1.7 & 6.2 & 1.9 & 3.1 & 3.0\end{array}$ summer

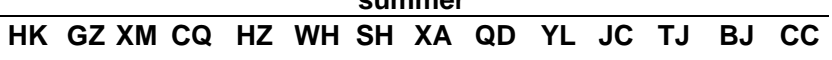
1.6

3.4

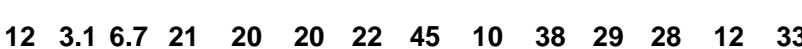

$\begin{array}{llllllllllllll}19 & 43 & 34 & 100 & 46 & 50 & 51 & 128 & 61 & 133 & 107 & 112 & 66 & 79\end{array}$ $\begin{array}{lllllllllllll}3.9 & 23 & 4.1 & 27 & 13 & 5.0 & 30 & 37 & & 3.5 & 12 & 51\end{array}$ $\begin{array}{llllllllllllll}17 & 23 & 25 & 38 & 31 & 34 & 23 & 30 & 4.1 & 8.3 & 1.2 & 38 & 21 & 45\end{array}$

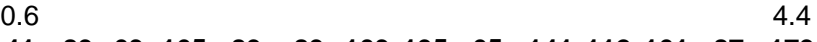
$\begin{array}{llllllllllllll}41 & 89 & 63 & 165 & 89 & 89 & 103 & 195 & 65 & 141 & 112 & 161 & 87 & 179\end{array}$

$\begin{array}{llllllllllll}0.4 & 0.8 & 4.7 & 0.8 & 1.6 & 1.9 & 0.2 & 0.6 & 0.1 & 0.5 & & 2.7\end{array}$ $\begin{array}{llllll}4.4 & 0.3 & 0.7 & 0.4\end{array}$

$\begin{array}{llllllllllllll}1.4 & 5.0 & 1.5 & 44 & 3.7 & 2.4 & 2.6 & 13 & 13 & 1.8 & 1.2 & 4.0 & 2.1 & 10\end{array}$ $\begin{array}{llllllllllllll}1.8 & 5.8 & 1.5 & 53 & 4.4 & 4.3 & 2.6 & 15 & 13 & 2.8 & 1.3 & 4.5 & 2.1 & 13\end{array}$

$9.5 \quad 3.0 \quad 4.5 \quad 13$

$$
\begin{aligned}
& \begin{array}{lllllllll}
11 & 4.0 & 5.8 & 1.0 & 81 & 2.2 & 6.3 & 4.3 & 4.6
\end{array} \\
& \begin{array}{llllll}
0.9 & 5.3 & 1.7 & 1.5 & 1.1
\end{array}
\end{aligned}
$$$$
\begin{array}{rr}
5.9 & 32 \\
3.0
\end{array}
$$$$
\begin{aligned}
& 3.0 \\
& 21
\end{aligned}
$$

$\begin{array}{llllllllllllll}9.5 & 3.0 & 10 & 68 & 24 & 4.0 & 20 & 1.0 & 81 & 7.2 & 19 & 5.5 & 20\end{array}$

$\begin{array}{llllllllllllll}0.2 & 1.1 & 0.8 & 3.1 & 1.9 & 0.3 & 1.8 & 2.3 & 0.5 & 1.3 & 1.6 & 1.2 & 1.0 & 0.6\end{array}$

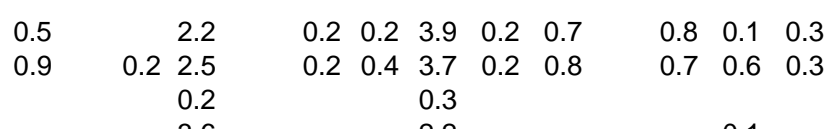

$\begin{array}{lllllllllllll}2.4 & 0.4 & 51 & 2.8 & 2.2 & 3.9 & 33 & 2.0 & 6.0 & 1.1 & 7.7 & 2.1 & 8.6\end{array}$ $\begin{array}{llllllllllllllll}0.3 & 0.1 & 8.1 & 0.2 & 0.6 & 0.6 & 4.6 & 0.3 & 0.8 & 0.1 & 1.3 & 0.1 & 1.3\end{array}$ $\begin{array}{llllllllllllll}0.1 & 0.6 & 0.1 & 15 & 0.8 & 1.0 & 0.9 & 9.6 & 0.5 & 1.6 & 0.4 & 1.8 & 0.8 & 2.3\end{array}$ $\begin{array}{lllllllllllll}0.1 & 11 & 0.2 & 0.1 & 0.2 & 4.7 & 0.1 & 0.9 & 0.9 & 0.3 & 0.9\end{array}$ $\begin{array}{lllllllll}0.2 & 2.4 & & 1.4 & 0.2 & 0.1 & 0.1 & 0.1\end{array}$ $\begin{array}{lllllllllllll}1.3 & 0.5 & 20 & 1.0 & 1.3 & 1.7 & 11 & 0.7 & 2.1 & 0.6 & 3.0 & 1.1 & 3.6\end{array}$ \begin{tabular}{llllll}
4.4 & & 3.1 & 0.4 & 0.1 \\
\hline
\end{tabular} 


\section{TABLE S-1 (Continued)}

benzo(ghi)perylene

anthanthrene

coronene

dibenzo(a,e)pyrene

subtotal

\section{Hopanes}

$17 \alpha(\mathrm{H})-22,29,30$-trisnorhopane $\left(\mathrm{C}_{27} \alpha\right)$

$17 \beta(\mathrm{H})-22,29,30$-trisnorhopane $\left(\mathrm{C}_{27} \beta\right)$

$17 \alpha(\mathrm{H}), 21 \beta(\mathrm{H})$-30-norhopane $\left(\mathrm{C}_{29} \alpha \beta\right)$

$17 \beta(\mathrm{H}), 21 \alpha(\mathrm{H})$-30-norhopane $\left(\mathrm{C}_{29} \beta \alpha\right)$

$17 \alpha(\mathrm{H}), 21 \beta(\mathrm{H})$-hopane $\left(\mathrm{C}_{30} \alpha \beta\right)$

$17 \beta(\mathrm{H}), 21 \alpha(\mathrm{H})$-hopane $\left(\mathrm{C}_{30} \beta \alpha\right)$

$17 \alpha(\mathrm{H}), 21 \beta(\mathrm{H})-22 \mathrm{~S}$-homohopane $\left(\mathrm{C}_{31} \alpha \beta \mathrm{S}\right)$

$17 \alpha(\mathrm{H}), 21 \beta(\mathrm{H})-22 R$-homohopane $\left(\mathrm{C}_{31} \alpha \beta \mathrm{R}\right)$

$17 \beta(\mathrm{H}), 21 \alpha(\mathrm{H})$-homohopane $\left(\mathrm{C}_{31} \beta \alpha\right)$

$17 \alpha(\mathrm{H}), 21 \beta(\mathrm{H})$-22S-bishomohopane $\left(\mathrm{C}_{32} \alpha \beta \mathrm{S}\right)$

$17 \alpha(\mathrm{H}), 21 \beta(\mathrm{H})-22 R$-bishomohopane $\left(\mathrm{C}_{32} \alpha \beta \mathrm{R}\right)$

subtotal

winter

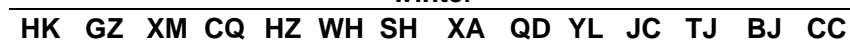

$\begin{array}{llllllllllllll}0.8 & 31 & 2.7 & 42 & 4.8 & 10 & 7.7 & 52 & 6.5 & 12 & 16 & 9.9 & 12 & 14\end{array}$ $\begin{array}{llllllllllllll}0.2 & 4.8 & 0.2 & 9.0 & & 1.1 & 16 & 0.8 & 2.1 & 1.0 & 1.5 & 3.3 & 4.2\end{array}$

$\begin{array}{lllllllllllllll}0.1 & 26 & 0.9 & 33 & 2.8 & 6.2 & 4.8 & 36 & 3.4 & 6.3 & 8.3 & 4.9 & 6.7 & 7.8\end{array}$ $\begin{array}{lllllllllll}7.7 & 0.6 & 29 & 5.8 & 22 & 1.6 & 2.4 & 12 & 1.6 & 3.4 & 3.1\end{array}$

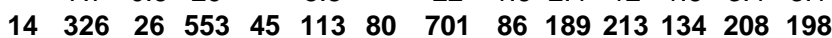

\section{$\begin{array}{lllllllllll}3.1 & 2.1 & 0.1 & 0.5 & 5.3 & 0.3 & 2.8 & 0.5 & 1.3 & 2.3 & 2.3\end{array}$} $\begin{array}{llllllllllll}0.9 & 0.2 & 0.3 & & 2.3 & 0.6 & 2.0 & 0.8 & 0.8 & 1.8 & 1.2\end{array}$ $\begin{array}{llllllllllllll}2.3 & 14 & 1.3 & 7.1 & 2.4 & 1.0 & 3.7 & 12 & 2.3 & 4.8 & 1.6 & 2.6 & 4.1 & 3.9\end{array}$ $\begin{array}{llllllllllll}0.1 & 2.0 & 0.1 & 6.0 & 0.7 & 3.4 & 1.1 & 1.4 & 3.6 & 2.1\end{array}$ $\begin{array}{lllllllllllllll}1.6 & 16 & 1.1 & 8.8 & 3.2 & 2.0 & 4.4 & 13 & 2.2 & 3.2 & 1.5 & 3.5 & 3.2 & 3.6\end{array}$ $\begin{array}{lllllllllllllll}16 & 1.1 & 8.8 & 3.2 & 2.0 & 4.4 & 13 & 2.2 & 3.2 & 1.5 & 3.5 & 3.2 & 3.6 \\ 1.6 & & 1.1 & & & 0.6 & 4.1 & 0.4 & 2.3 & 0.7 & 0.9 & 1.9 & 1.6\end{array}$ $\begin{array}{lllllllllllll}8.7 & 0.4 & 3.8 & 0.8 & 0.7 & 1.8 & 4.8 & & 0.8 & 0.3 & 0.7 & 1.2 & 1.5\end{array}$ $\begin{array}{llllllllllll}5.1 & 0.3 & 2.2 & 0.5 & 1.0 & 3.4 & 0.8 & 0.4 & 0.8 & 1.2 & 1.0\end{array}$ $\begin{array}{llllllll}0.3 & 1.2 & 0.8 & 0.3 & 0.2 & 0.1 & 0.8\end{array}$

$\begin{array}{llllllllll}4.4 & 2.0 & 1.5 & 1.2 & 0.6 & 0.2 & 0.3 & 0.7 & 0.6\end{array}$

$\begin{array}{lllllllll}4.1 & 1.3 & 0.8 & 1.5 & 0.4 & 0.2 & 0.5 & 0.7\end{array}$

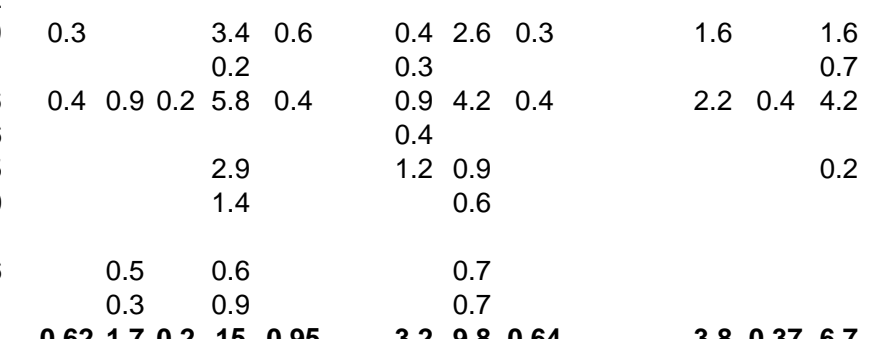

$\begin{array}{lllllllllllllllllllllllll}4.1 & 60 & 3.2 & 29 & 7.3 & 3.6 & 15 & 54 & 6.5 & 22 & 7.5 & 13 & 22 & 18 & 0.62 & 1.7 & 0.2 & 15 & 0.95 & 3.2 & 9.8 & 0.64 & 3.8 & 0.37 & 6.7\end{array}$

${ }^{a}$ City code: HK (Hong Kong), GZ (Guangzhou), XM (Xiamen), CQ (Chongqing), HZ (Hangzhou), WH (Wuhan), SH (Shanghai), XA (Xi'an), QD (Qingdao), YL (Yulin), JC (Jinchang),

TJ (Tianjin), BJ (Beijing) and CC (Changchun);

${ }^{\mathrm{b}} \mathrm{CPI}$, carbon preference index: $\left(\mathrm{C}_{17}+\mathrm{C}_{19}+\mathrm{C}_{21}+\cdots \cdots+\mathrm{C}_{35}\right) /\left(\mathrm{C}_{16}+\mathrm{C}_{20}+\mathrm{C}_{22}+\cdots \cdots+\mathrm{C}_{34}\right)$ for $n$-alkanes and $\left(\mathrm{C}_{10}+\mathrm{C}_{12}+\mathrm{C}_{14}+\cdots \cdots+\mathrm{C}_{34}\right) /\left(\mathrm{C}_{9}+\mathrm{C}_{11}+\mathrm{C}_{13}+\cdots \cdots+\mathrm{C}_{35}\right)$ for fatty acids $(1)$;

(1) Simoneit, B. R. T.; Kobayashi, M.; Mochida, M.; Kawamura, K.; Lee, M.; Lim, H. J.; Turpin, B. J.; Komazaki, Y. Composition and major sources of organic compounds of aerosol particulate matter sampled during the ACE-Asia campaign. J. Geophys. Res. 2004, 109 (D19S10), doi:10.1029/2004JD004598. 
(a) Fatty alcohols (m/z 75)

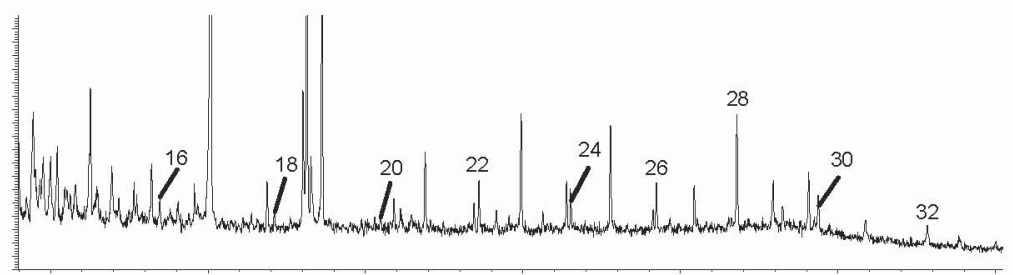

(b) Polyols and polyacids ( $\mathrm{m} / \mathrm{z} 147)$

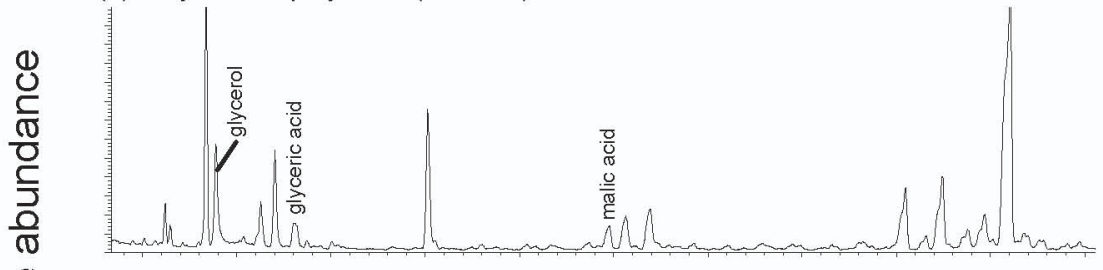

(c) Sterols ( $\mathrm{m} / \mathrm{z}$ 129)

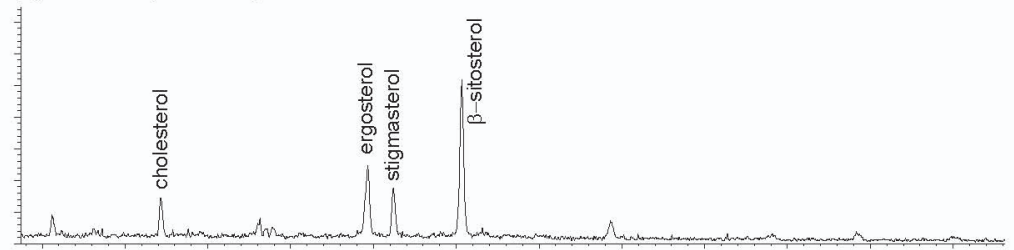

(d) Hopanes ( $\mathrm{m} / \mathrm{z} 191)$

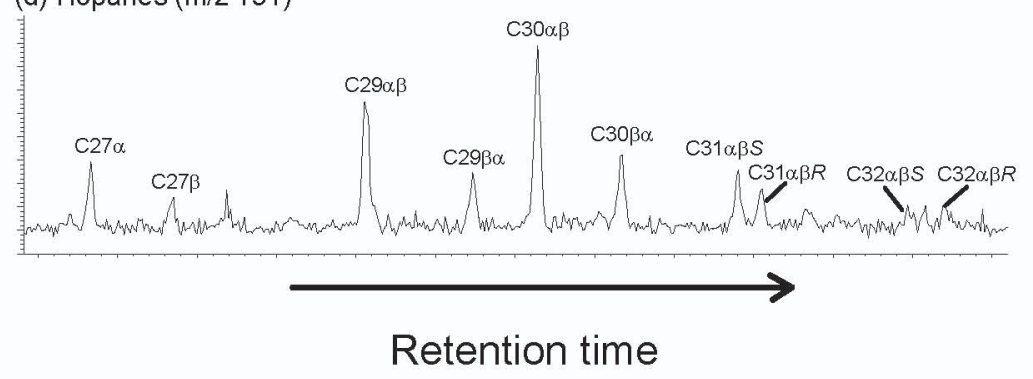

Figure S-1. Salient features of the GC/MS traces for the minor compounds in aerosls from Xi'an during winter (01/13/2003) (a) mass chromatogram (m/z 75) of fatty alcohols, (b) mass chromatogram (m/z 147) of polyols and polyacids, (c) mass chromatogram (m/z 129) of sterols, and (d) mass chromatogram ( $\mathrm{m} / \mathrm{z} 191)$ of hopanes. 